\title{
Multilayer Structure Technique for Improving Determination of Electromagnetic Properties of Radar Absorbers Based on A Two-Layer Method and Flanged Rectangular Waveguide Probe
}

\author{
Abdulkadhim A. Hassan ${ }^{1}$, Janan H. Saadie ${ }^{2}$ \\ ${ }^{1,2}$ Department of Electrical Engineering, Kufa University, Iraq
}

\section{Article Info}

Article history:

Received Jul 20, 2019

Revised Oct 27, 2020

Accepted Oct 29, 2020

\section{Keywords:}

Layered structure

FDTD

Constitutive parameters

Rectangular waveguide

Microwave measurement

\begin{abstract}
This paper presents further development of utilization of a two-layer method to perform nondestructive electromagnetic properties determination of planar radar absorbers using flanged open-ended rectangular waveguide probe. A multilayer structure of three layers was proposed to improve the measured results of these parameters obtained using two-layer method. These layers were arranged such that the test material is sandwiched between two known low loss materials to provide the two needed independent reflection coefficients necessary to extract them at different conditions of testing. The proposed structure was aimed to decrease the effect of direct backing of test material by metal plate, which influences measurement accuracy if two-layer method is used. The structure permits a suitable electric field interrogation in test material and decreases the influences of both radial and surface waves. FDTD method was adapted for modeling the problem geometry to calculate the reflection coefficients since a probe with finite flange size is used. Measurements were carried out using the proposed technique to determine complex permittivity and complex permeability of several radar absorbers over X-band applications of microwaves. In comparison with both single-layer and two-layer methods results, the measured results of these parameters agreed well with the published data by companies and literatures.
\end{abstract}

Copyright $@ 2020$ Institute of Advanced Engineering and Science. All rights reserved.

Corresponding Author:

Abdulkadhim A. Hassan,

Department of Electrical Engineering,

Kufa University,

Kufa Road, Al-Najaf Governorate, Iraq.

Email: abdulkadhim.shlash@uokufa.edu.iq

\section{INTRODUCTION}

In many applications of microwaves, evaluation of materials electromagnetic properties accurately has significant importance over the recent decades. Examples are applications of radar absorbers in electromagnetic shielding, radar stealth and electromagnetic compatibility [1-4]. Based on electromagnetic field interaction with materials, researchers have spent considerable efforts, for many years, in developing measurement techniques to measure both complex permittivity $\left(\varepsilon_{\mathrm{r}}\right)$ and complex permeability $\left(\mu_{\mathrm{r}}\right)$ conveniently, accurately and quickly [5-8]. Each one of these techniques is suitable for some specific applications and has some distinct advantages and drawbacks. In comparison with the other methods, the technique using rectangular waveguide probe with open-end is well suited for nondestructive in situ solid materials characterization. The restrictions for diminishing of sample preparation, the openness property of the probe structure and ability of handling of high power are the main reasons. Moreover, the reflection property of the probe $(\Gamma)$ is sensitive to more than one variable, which allows to measure different parameters of test material such as electromagnetic parameters $\left(\varepsilon_{r}, \mu_{r}\right)$ and its thickness as well [9-13].

To simultaneously determine both $\varepsilon_{\mathrm{r}}$ and $\mu_{\mathrm{r}}$ parameters using open-ended rectangular waveguide probe, it needs to measure two reflection coefficients obtained under different conditions of testing. Most commonly these parameters are numerically extracted by inverse problem from the measured $\left(\Gamma_{\text {meas }}\right)$ and 
theoretically calculated $\left(\Gamma_{\text {thy }}\right)$ reflection coefficients using iterative search algorithms. To achieve this purpose, the probe terminated by test material is analytically modeled using different approaches to theoretically formulate its reflection coefficient. In all of these formulations, the probe flange is assumed to be infinitely large, which cannot be physically realized in practice. This assumption leads to approximate solution of theoretical reflection coefficient compared to the measured one since probe with definite flange dimension is used. This assumption introduces uncertainty in the extracted $\varepsilon_{\mathrm{r}}$ and $\mu_{\mathrm{r}}$ results

The reflection coefficient measured using open-ended rectangular waveguide probe is a complex function of several variables. These variables are, probe dimension $(a$ and $b)$, frequency of measurement $(f)$, test material thickness $(d)$ and electromagnetic properties $\left(\varepsilon_{r}\right.$ and $\left.\mu_{r}\right)$, symbolized as $\Gamma_{\text {meas }}\left(a, b, f, d, \varepsilon_{\mathrm{r}}, \mu_{\mathrm{r}}\right)[9]$. Based on the given function of probe reflection coefficient, three techniques have been developed for a given probe dimension to independently measure the required two reflection coefficients. These techniques can be performed via changing thickness of the test material $(d)$, or changing the measurement frequency $(f)$, or changing part of the tested material. They are respectively called Thickness-Varying Method (TVM) [11], Frequency-Varying Method (FVM) [12] and Sample-Varying Method (SVM) [11]. Both TVM method and FVM method are performed using single-layer (test material only) while SVM method is performed using two layers of known material followed by test material. Each one of these methods has its own advantages and drawbacks depending on requirements of the measurement. For example, in FVM method, the frequency is taken to be independently varied so that the two reflection coefficients are measured using one sample at two adjacent frequencies $\left(f_{1}\right.$ and $\left.f_{2}\right)$. This method is suitable to be used for broadband and single frequency material characterization. When it is needed to test a material fabricated as designed in the laboratory environment, TVM proves to be more suitable and accurate method. It makes two reflection measurements with two samples of different thickness. However, For in-situ measurement situations, SVM method or twolayer method is the viable option than TVM since the measurement of $\Gamma_{\mathrm{m}}$ is performed on one sample to test first, then a combination of the same sample with another one of the known, $\varepsilon_{r}$ and $\mu_{r}$ to be tested again, to get two reflection coefficients. The obtained structure forms a medium with two layers. Consequently, it is called a two-layer method. This method is used to determine both $\varepsilon_{r}$ and $\mu_{r}$ of radar absorbers using a rectangular waveguide probe with the flange assumed to be infinite in extension [7-9][11]. In the aforementioned methods, the test material is usually backed by metal plate to increase the measurement sensitivity. Researches have shown that the accuracy of high-loss materials $\varepsilon_{r}$ and $\mu_{r}$ measurements using a two-layer method is highly influenced by the presence of backing metal plate [7-8]. This is due to that backing test material by metal plate leads to existence of small electric field interrogation in test material. On the other hand, the existence of the surface and radial radiation waves excited within the metal plate and the flange bounded area would give rise to a serious problem in the measurement results. Therefore, this method has to be further investigated and studied to solve these problems. In this paper, a multilayer structure technique was proposed to improve $\varepsilon_{r}$ and $\mu_{r}$ parameters measurement accuracy of high-loss materials obtained using the existing two-layer method. The structure was employed using three layers backed by a metal plate and irradiated by a finite size flange rectangular waveguide probe. The three layers are organized such that the test material with known thickness and unknown EM parameters is sandwiched between two known low-loss materials. This arrangement of the three layers permits to produce a considerable electric field into test material. Both $\varepsilon_{\mathrm{r}}$ and $\mu_{\mathrm{r}}$ are to be extracted from measurement of two reflection coefficients performed under two physical conditions of testing. The first one is obtained when the test material is sandwiched between two identical layers of low-loss material while the second one is obtained when the test material is sandwiched between two different layers of low-loss materials or vice versa depending on the nature of known materials used for this purpose. The proposed structure is aimed to overcome the influence of direct backing of the test material by a metal plate as well as reducing the influence of radial and surface waves that may exist in the open boundary of the problem if the two-layer method is used. The FDTD method was employed to numerically predict probe reflection coefficient due to using probe with flange of finite size, where employing the analytical approaches in such situations is quite difficult. An analysis was made to evaluate the proposed technique performance compared to the existing methods (both single-layer and two-layer methods). The measured results of $\varepsilon_{\mathrm{r}}$ and $\mu_{\mathrm{r}}$ for selected samples of radar absorbers are to be compared with the reference data and data published by literatures to validate the feasibility of the proposed technique.

\section{THE PROPOSED MEASUREMENT TECHNIQUE}

In principle, to perform multi-parameter measurement, the mathematical truth associated with this process states that in order to determine the values of $n$ unknowns, it needs to independently perform $n$ measurements in order to obtain all or even any one of these unknowns. Therefore, to determine both $\varepsilon_{\mathrm{r}}$ and $\mu_{\mathrm{r}}$ parameters, two independent complex reflection coefficients are needed to be measured at two different 
conditions of testing. As mentioned before, they can be obtained using either single-layer method or twolayer method. Figure 1(a) illustrates a single-layer method geometry and Fig. 1(b) illustrates the geometry of the two-layer method. For single-layer method, either FVM or TVM can be employed on the same sample to obtain the two needed reflection coefficients. For two-layer method, the first reflection coefficient is obtained by performing a measurement using only the test material first while the second one is obtained by placing the probe against a combination of known low-loss material followed by test material. In both methods, backing of test material by metal plate yields an accurate result of $\mu_{\mathrm{r}}$ measurement, but there is a difficulty in accurately determining of $\varepsilon_{\mathrm{r}}$. This is due to forcing the transverse electric field to zero at metal plate walls. Consequently, a small electric field interrogates with test material resulting in inaccurate measurement of $\varepsilon_{\mathrm{r}}$ while for the magnetic field, the opposite is true. Intuitively, the complement of metal plate backing is obtained in the measurement of $\varepsilon_{\mathrm{r}}$ and $\mu_{\mathrm{r}}$ when the free space backing is used instead. In this case, the electric field with a relatively large value is permitted resulting in accurate results of $\varepsilon_{\mathrm{r}}$ while a magnetic field with small value exists in the test material yielding in inaccurate $\mu_{\mathrm{r}}$ results. On the other hand, the radiated waves and the surface waves that may exist in the open boundary space of the problem would give rise to considerable influence on both $\varepsilon_{\mathrm{r}}$ and $\mu_{\mathrm{r}}$ measurement. In order to improve measurement accuracy, a threelayer structure backed by a metal plate is proposed in conjunction with FDTD method to achieve this purpose. The proposed structure consists of a combination of two layers of low-loss materials each with known EM parameters and thickness and one layer of test material of known thickness and unknown $\varepsilon_{\mathrm{r}}$ and $\mu_{\mathrm{r}}$ sandwiched between them.

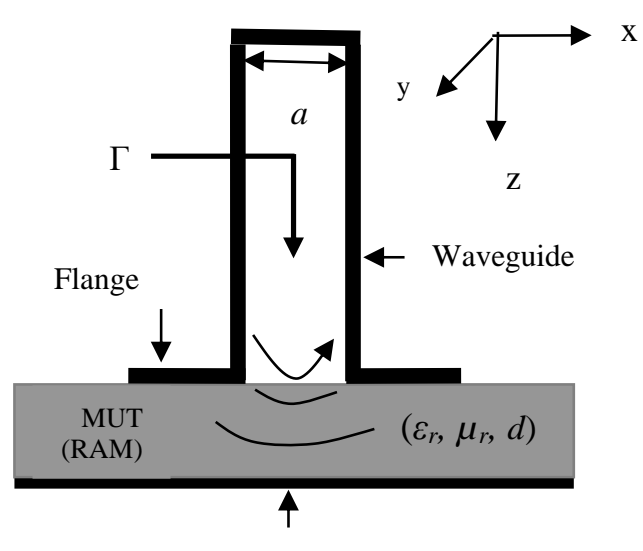

Metal Plate

(a)

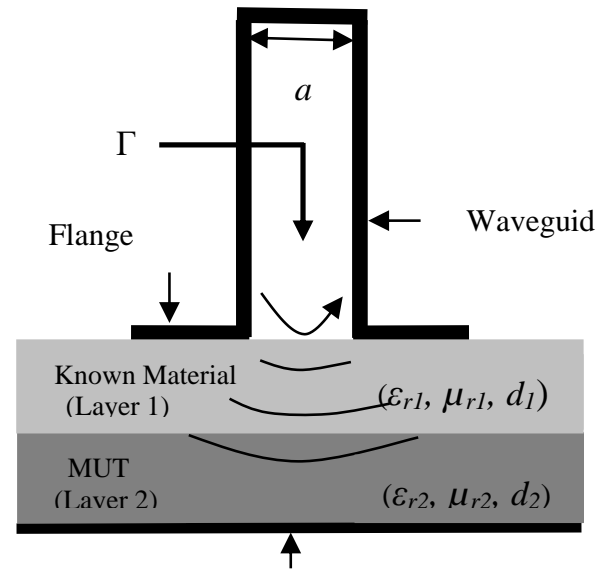

Metal Plate

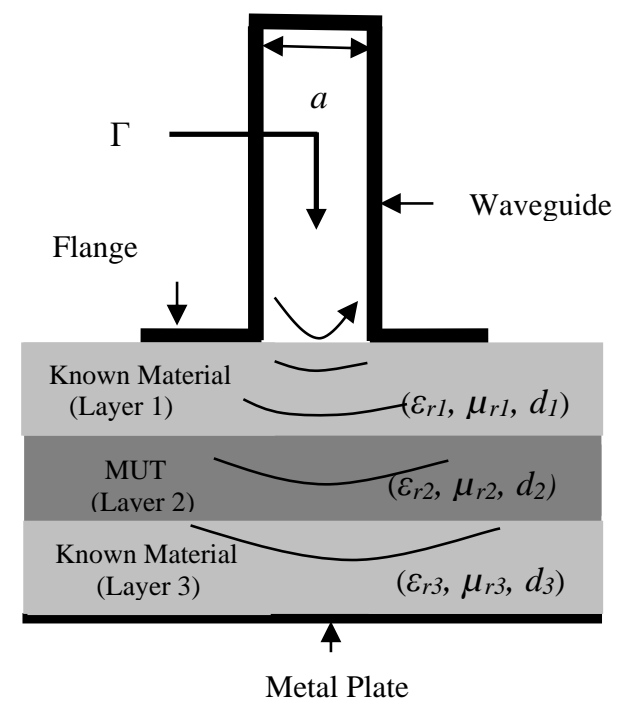

(b)

(c)

Figure 1. The configurations of the different methods used for materials characterization (a) Single-layer method (b) Two- layer method (c) The proposed technique. 
The whole structure is irradiated by X-band rectangular waveguide probe placed against the structure as depicted in Fig. 1 (c). As illustrated in the figure, the third layer of known material is used to decrease the influence of direct backing of the test material by metal plate while the first layer of known material is used to decrease the influence of the radial and the surface waves that may exist at the open boundary of the problem between probe flange and test material. This arrangement permits a reasonable interrogating of electric field with the test material compared to the case of direct backing by metal plate using two-layer method. Consequently, a considerable improvement in both $\varepsilon_{\mathrm{r}}$ and $\mu_{\mathrm{r}}$ measurement accuracy can be obtained.

Using the proposed technique, the measured two independent reflection coefficients $\left(\rho_{1}\right.$ and $\left.\rho_{2}\right)$ are obtained in two steps. The first one $\left(\rho_{1}\right)$ is obtained when the test material is sandwiched between two identical layers of low-loss material while the second one $\left(\rho_{2}\right)$ is obtained when the test material is sandwiched between two different layers of low-loss materials. The measurement process is described by the following set of simultaneous equations:

$$
\begin{gathered}
\rho_{1}=\Gamma_{\text {meas }}\left(f, d_{1}, d_{2}, d_{3}, \varepsilon_{r 1}, \mu_{r 1}, \varepsilon_{r 2}, \mu_{r 2}, \varepsilon_{r 1}, \mu_{r 1}\right) \\
\rho_{2}=\Gamma_{\text {meas }}\left(f, d_{1}, d_{2}, d_{3}, \varepsilon_{r 1}, \mu_{r 1}, \varepsilon_{r 2}, \mu_{r 2}, \varepsilon_{r 3}, \mu_{r 3}\right)
\end{gathered}
$$

Where $f$ is the frequency of measurement and Fig.1(c) defines the other symbols pictorially. The two known materials should be selected so that the two measured reflection coefficients will contain at least partly different information and can be distinguished by a network analyzer. From (1) and (2) both $\varepsilon_{\mathrm{r}}=\left(\varepsilon^{\prime}-\right.$ $\left.j \varepsilon^{\prime \prime}\right)$ and $\mu_{\mathrm{r}}=\left(\mu^{\prime}-j \mu^{\prime \prime}\right)$ of test material can be extracted by finding the roots of the two functions $\mathrm{f}\left(\varepsilon_{\mathrm{r}}, \mu_{\mathrm{r}}\right)$ and $\mathrm{g}\left(\varepsilon_{\mathrm{r}}, \mu_{\mathrm{r}}\right)$ through numerical iterations by which the difference between the measured and theoretical $\left(\Gamma_{\text {thy }}\right)$ reflection coefficients obtained at different test conditions is minimized using (3):

$$
\left\{\begin{array}{l}
f\left(\varepsilon_{r}, \mu_{r}\right)=\Gamma_{1}^{\text {meas }}-\Gamma_{1}^{\text {thy }} \\
g\left(\varepsilon_{r}, \mu_{r}\right)=\Gamma_{2}^{\text {meas }}-\Gamma_{2}^{\text {thy }}
\end{array}\right.
$$

It is apperent from (3) that accurate extraction of $\varepsilon_{\mathrm{r}}$ and $\mu_{\mathrm{r}}$ depends on having accurate knowledge of theoretically developed probe reflection coefficient. Additional error may occur when $\Gamma_{\text {thy }}$ with approximate solution is used. Thus, the reflection coefficient is numerically formulated using FDTD method to obtain better approximation since employing the analytical methods in this situation is quite difficult.

\section{NUMERICAL FORMULATION OF THE PROBLEM}

\subsection{Reflection Coefficient Calculation using FDTD Method}

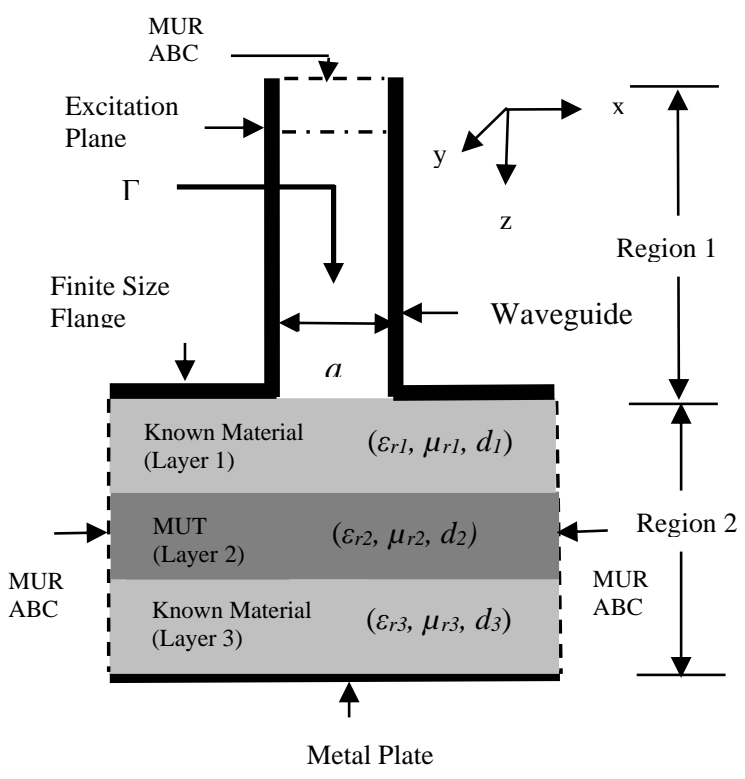

Figure 2. The FDTD computational model of the problem 
In this work, the reflection coefficient $(\Gamma)$ seen by probe aperture is numerically calculated using FDTD modelling of the actual probe/multilayer structure geometry as depicted in Fig. 2. The rectangular waveguide probe is used with dimensions, $a$ and $b$, chosen to propagate the dominant mode ( $\mathrm{TE}_{10}$ ) only in the $+\mathrm{z}$ direction at $\mathrm{X}$-band range of microwaves. The reflected wave from the layered structure carries the desired information of the test material $\varepsilon_{\mathrm{r} 2}$ and $\mu_{\mathrm{r} 2}$ within the layered structure. The available space of the FDTD computational domain shown in Fig. 2 has two regions. The probe interior region (region 1) surrounded by waveguide walls which are assumed to be perfect conductors. The second region (region 2) includes the layered structure backed by a perfectly conducting metal plate. The layered-structure consists of the test material layer with unknown EM parameters and known thickness sandwiched between two layers of known low-loss material. Under the assumption that the media of the two regions are linear, homogeneous and isotropic, the interaction between the electromagnetic (EM) field and the different media of the problem are described by Maxwell's curl equations using (4) (6):

$$
\begin{aligned}
& \frac{\partial D}{\partial t}=(\nabla x H) \\
& D=\varepsilon_{0} \cdot \varepsilon_{r} \cdot E \\
& \frac{\partial H}{\partial t}=\frac{1}{\mu_{o} \mu_{r}}(\nabla x E)
\end{aligned}
$$

Where $D$ is the density of the electric flux, $E$ and $H$ are the electromagnetic fields intensities respectively and $\varepsilon_{\mathrm{r}}$ and $\mu_{\mathrm{r}}$ are EM parameters of the respective medium in each region. The normalized equation of $(4) \sim(6)$ can be written as:

$$
\begin{aligned}
& \frac{\partial D}{\partial t}=\frac{1}{\sqrt{\varepsilon_{o} \mu_{o}}}(\nabla x H) \\
& D=\varepsilon_{r} \cdot E \\
& \frac{\partial H}{\partial t}=\frac{1}{\sqrt{\varepsilon_{o} \mu_{o}} \mu_{r}}(\nabla x E)
\end{aligned}
$$

The FDTD method proposed by Yee [14] is a technique that numerically solves (4) (6) in time domain. It gives the evaluation of the fields in time for giving known excitation. Following Yee procedure, the vector equations (4) and (6) are translated into a six scalar first-order set of partial differential equations in $\mathrm{x}, \mathrm{y}$ and $\mathrm{z}$ coordinates. For $\mathrm{x}$ coordinate, the $\mathrm{E}$ and the $\mathrm{H}$ field's components, are given using (10) and (11):

$$
\begin{aligned}
& \frac{\partial E_{x}}{\partial t}=\frac{1}{\varepsilon_{r} \sqrt{\varepsilon_{o} \mu_{o}}}\left(\frac{\partial H_{z}}{\partial y}-\frac{\partial H_{y}}{\partial z}\right) \\
& \frac{\partial H_{x}}{\partial t}=\frac{-1}{\mu_{r} \sqrt{\varepsilon_{o} \mu_{o}}}\left(\frac{\partial E_{y}}{\partial z}-\frac{\partial E_{z}}{\partial y}\right)
\end{aligned}
$$

The same procedure is applied using (4) and (6) to obtain the other fields components for y and $\mathrm{z}$ coordinates. The six scalar equations of (7) and_(9) represent the FDTD method basis. The Maxwell's curl equations of (4) and (6) are discretized using FDTD method in both time and spatial domains directly with dividing the computational domain into Yee unit cells. Using 3-D Cartesian coordinate system, the available FDTD space is divided into a lattice structure of a cubic shape with dimensions of $\Delta x$ in $x$-axis, $\Delta y$ in $y$-axis and $\Delta \mathrm{z}$ in z-axis. Applying both space and time central difference approximations to (10) and (11) gives the time and space second order accuracy using (12) and (13).

$$
\begin{aligned}
& E_{x}^{n+1}(\mathrm{i}+1 / 2, \mathrm{j}, \mathrm{k})=E_{x}^{n}(\mathrm{i}+1 / 2, \mathrm{j}, \mathrm{k}) \\
& \quad+\frac{\Delta t}{\Delta y \cdot \varepsilon_{r} \cdot \sqrt{\varepsilon_{o} \mu_{o}}}\left(H_{z}^{n+1 / 2}(i+1 / 2, j+1 / 2, k)-H_{z}^{n+1 / 2}(i+1 / 2, j-1 / 2, k)\right) \\
& \quad-\frac{\Delta t}{\Delta z \cdot \varepsilon_{r} \cdot \sqrt{\varepsilon_{o} \mu_{o}}}\left(H_{y}^{n+1 / 2}(i+1 / 2, j, k+1 / 2)-\left(H_{y}^{n+1 / 2}(i+1 / 2, j, k-1 / 2)\right)\right.
\end{aligned}
$$




$$
\begin{aligned}
& H_{x}^{n+1 / 2}(\mathrm{i}, \mathrm{j}+1 / 2, \mathrm{k}+1 / 2)=H_{x}^{n-1 / 2}(\mathrm{i}, \mathrm{j}+1 / 2, \mathrm{k}+1 / 2) \\
& \quad+\frac{\Delta t}{\Delta y \cdot \mu_{r} \cdot \sqrt{\varepsilon_{o} \mu_{o}}}\left(\mathrm{E}_{z}^{n}(i, j+1, k+1 / 2)-E_{z}^{n}(i, j, k+1 / 2)\right) \\
& \quad-\frac{\Delta t}{\Delta z \cdot \mu_{r} \cdot \sqrt{\varepsilon_{o} \mu_{o}}}\left(\mathrm{E}_{y}^{n}(i, j+1 / 2, k+1)-E_{y}^{n}(i, j+1 / 2, k)\right)
\end{aligned}
$$

In FDTD method, one cell distance of wave propagation requires a time step with a minimum value determined by the Courant condition in 3-D simulation using (14).

$$
\Delta t \leq \frac{1}{\mathrm{c}\left[\frac{1}{\Delta x^{2}}+\frac{1}{\Delta y^{2}}+\frac{1}{\Delta z^{2}}\right]^{1 / 2}}
$$

Where $c$ is light velocity. To satisfy the Courant condition of the algorithm stability of the FDTD method, $\Delta$ is to be calculated under the condition of $\lambda / 10$ where $\lambda$ is the maximum frequency wavelength. To truncate the problem's computational space as well as increase the computing efficiency, the first-order approximately boundary conditions are applied [15] in the radial boundary space of the problem geometry. Using the first-order approximately boundary conditions is adequate due to that the probe radiation is limited and its ability to truncate the boundaries with discontinuity of dielectric properties of the different layers. The calculation of the probe reflection coefficient is performed using a pulse with Gaussian derivative [16] incident normally on the structure. The feeding of the probe is made from excitation plane located sufficiently far from the aperture. The sampling point of the fields is located a away a distance from the aperture for the higher order modes to be avoided. Both permittivity and permeability are used with their averaged values in the boundary interfaces between different layers to reduce the errors that may occur due to using different materials in the structure. The reflection coefficient $(\Gamma)$ is calculated using (15):

$$
\Gamma=\frac{Y_{a}-Y_{0}}{Y_{a}+Y_{0}}
$$

Where $Y_{a}$ is the admittance of the aperture and $Y_{0}$ is the waveguide equivalent characteristic admittance.

\subsection{Numerical Testing}

The presented FDTD formulation of the problem was applied in computer routines to calculate probe complex reflection coefficients at different test conditions using a code developed for this purpose. To verify and validate the accuracy of the developed FDTD code, simulation and experiments were carried out on radar absorbing material using WR-90 X-band rectangular waveguide probe to calculate and measure reflection coefficient $(\Gamma)$ seen by probe aperture. In this work, a fine space cells were used for the probe space and the layered structure to increase the calculation accuracy. Table 1 lists the used parameters of the FDTD simulation. The FDTD calculated results of reflection coefficient (magnitude and phase) were compared first with experimental results $\left(\Gamma_{\text {meas }}\right)$ and then with the results obtained analytically using the formulation previously developed for determination of probe reflection coefficient $\left(\Gamma_{\text {thy }}\right)$ of multilayer medium [11]. In this regard, two cases were considered: the first test was made using a single-layer method to calculate the probe reflection coefficient terminated by GEC-Marconi RAM-9052 absorbing material with constitutive parameters of $\varepsilon_{\mathrm{r}}=18.18-j 0.418$ and of $\mu_{\mathrm{r}}=1.55-j 1.984$ and $2.08 \mathrm{~mm}$ thickness. The second test was made using a twolayer method with the layers organized such that the probe is placed in close contact with Teflon as known material (layer 1) followed by GEC-9052 test material as the second layer. In both cases, the test material is

Table 1. The FDTD simulation parameters

\begin{tabular}{cccc}
\hline Number of Cell & size of Cell & Time step & number of Steps \\
\hline \multirow{3}{*}{304000} & $\Delta \mathrm{x}=0.517 \mathrm{~mm}$ & $1 \times 10^{-12} \mathrm{sec}$ & 45000 \\
& $\Delta \mathrm{y}=0.508 \mathrm{~mm}$ & & \\
$\Delta \mathrm{z}=0.60 \mathrm{~mm}$ & & \\
\hline
\end{tabular}


backed by a metal plate with $8 \mathrm{~mm}$ thickness of Teflon layer. The experiments were carried out using network analyzer (ANA HP-8510B) and the results are shown in table 2. It is clear from the table that for the two cases under study, the variations in the reflection coefficients (both magnitude and phase) obtained experimentally are fairly consistent with the simulation results of the FDTD method. The obtained results validate the used tool of the computation.

\section{Numerical Analysis}

In this paper, a three-layer structure is utilized in order to improve measurement accuracy of $\varepsilon_{\mathrm{r}}$ and $\mu_{\mathrm{r}}$ of high loss material instead of using two-layer method. As mentioned before, one of these three layers is test material layer sandwiched between two layers of materials with known EM parameters and thicknesses. In this regard, the selection of the two known materials is a critical task in the measurement process using the proposed technique to provide the conditions for obtaining two independent reflection coefficients necessary to extract them. On the other hand, the two known materials, must be selected with a specific EM parameters and thickness in order to enhance the sensitivity of reflection property measurement. Consequently, low-loss materials with different loss factors should be selected to ensure that the two measured reflection coefficients are partly carrying different information and can be distinguished by network analyzer. Among the commercially available low-loss materials, both Teflon and Acrylic are chosen to be used as known materials. These two materials are with low-dispersion and rigid. Also, the loss factor of Acrylic is slightly higher than that of Teflon making them suitable to be used for this purpose. Based on the literatures [17], the complex parameters for both Teflon and Acrylic are $\varepsilon_{\mathrm{r}}=2.04-j 0.007$ and $\mu_{\mathrm{r}}=1-j 0.0$ and $\varepsilon_{\mathrm{r}}=2.61-$ $j 0.012$ and $\mu_{\mathrm{r}}=1-j 0.0$ respectively. A numerical analysis was performed to investigate the influences of both flange size of the probe and known materials layers thicknesses on the measured reflection coefficient for different thickness of test material as described in the following subsections.

\subsection{Influence of Probe Flange size}

As mentioned before, one of the error sources in the extracting of $\varepsilon_{\mathrm{r}}$ and $\mu_{\mathrm{r}}$ of tested material using rectangular waveguide probe with the open end resides with the flange of finite size. In previous studies, either assumption of very large flange size was made [9] or it is chosen based on the aperture electric field pattern [11]. Practically, the probe flange is finite in size. To investigate flange size influence on the measured probe reflection coefficient, FDTD method was employed to perform 3-D simulations for the case when only the test material is considered as shown in Fig. 1(a). The test material used in the analysis is Eccosorb MF-116 radar absorbing material with constitutive parameters of $\varepsilon_{\mathrm{r}}=16-j 0.96$ and $\mu_{\mathrm{r}}=1.5-j 1.02$. The probe with different thicknesses of test material was simulated using WR-90 rectangular waveguide to calculate the deviation (in \%) of measured probe reflection coefficient as the probe flange size varies from the minimal value of the flange to the largest considered value at frequency of $10 \mathrm{GHz}$. The obtained results were compared with the results obtained when a probe with an infinite size of a flange is used. Fig. 3 shows the deviation (in \%) of the calculated reflection coefficient versus the flange size variation for three selected

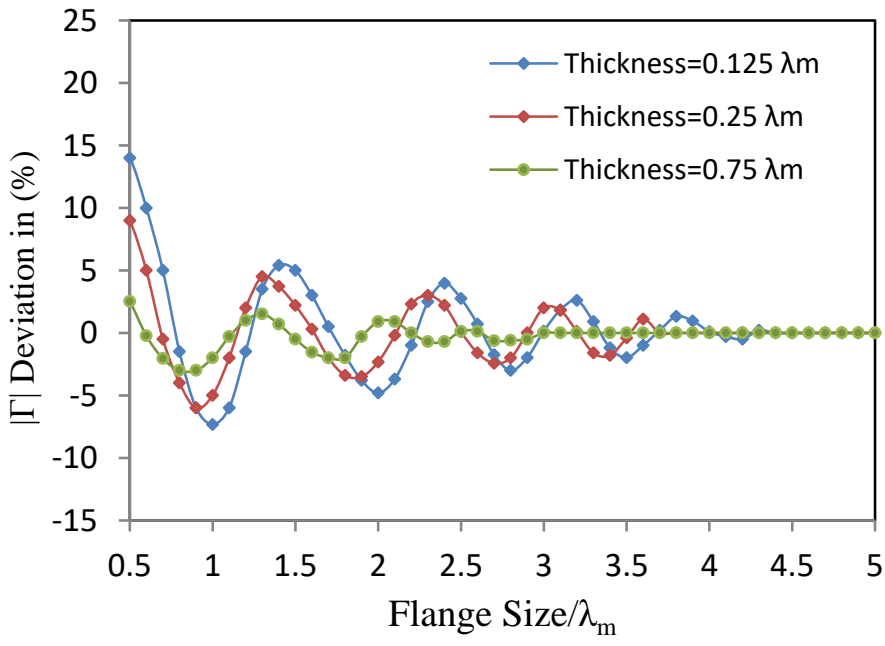

Figure 3. Variation of probe reflection coefficient with the normalized flange size for lossy material of different thickness. 
cases of test material thickness of $0.125 \lambda_{\mathrm{m}}, 0.25 \lambda_{\mathrm{m}}$ and $0.75 \lambda_{\mathrm{m}}$ respectively. The percentage deviation in $|\Gamma|$ is defined as:

$$
\% \text { Deviation }|\Gamma|=100 x \frac{\left|\Gamma_{\mathrm{inf}}-\Gamma_{\text {fin }}\right|}{\left|\Gamma_{\mathrm{inf}}\right|}
$$

where $\lambda_{\mathrm{m}}$ is the wavelength in the test material calculated at maximum frequency of $\mathrm{X}$-band, $\Gamma_{\mathrm{Inf}}$ is the probe reflection coefficient calculated using spectral domain analysis model [18] developed for the probe with flange assumed to be infinitely large, and $\Gamma_{\mathrm{Fin}}$ is the finite size of the flange reflection coefficient calculated using FDTD method. In the figure the influence of test material thickness on the calculated reflection coefficient is obvious. The effect of the size of probe flange on variation of reflection coefficient in case of the thick sample is less compared to that of the thin sample. For high loss materials, this is reasonable since the reflection property decreases with increasing of test material thickness. Also, for a given sample thickness, the reflection coefficient variation decreases with increasing of flange size. This is due to the fact that for small flange size, the flange finite edge termination of the material layer causes spurious signals reflected from the boundaries. The reflection of spurious signals decreases with increasing of flange size. It is clear from the figure that for the $0.75 \lambda_{\mathrm{m}}(6 \mathrm{~mm})$ sample thickness, the variation in reflection coefficient magnitude for this sample becomes duller, which in turn tends to be point as flange size increases. This is due to the fact that the test material is so thick that the wave of reflection decaying from the metal plate has no influence on the input wave at the plane of the flange. On the other hand, ensuring accuracy in measurement becomes quite difficult if the test material is chosen to be with thin thickness. The thin thickness of test material leads to a high value of the measured probe reflection coefficient. The obtained results show that a flange size with dimension larger than $\left(\lambda_{\mathrm{o}}\right)$ is sufficient for reflection coefficient of a thick lossy material to be accurately measured since the caused error in this case is very small. However, a flange with a relatively large dimension is required $\left(\geq 2 \lambda_{\mathrm{o}}\right)$ for thin and lossy test material in order to obtain an accurate reflection coefficient measurement. Thus, choosing a proper material thickness for testing is necessary to obtain accurate results. For the proposed technique, it was found that the optimum range for test material thickness is between $1 \mathrm{~mm}$ and $5 \mathrm{~mm}$. For the examined cases, the variation of probe reflection coefficient with probe flange size had an oscillatory-damped dependence.

\subsection{Accessing The Known Materials Thickness}

A numerical analysis was performed using FDTD method to access the influence of the known materials thickness of the layered structure on magnitude variation of probe reflection coefficient. In this analysis, four cases of the layered structure were considered using Eccosorb MF-116 radar absorbing material as a test material. The probe used is X-band WR-90 rectangular waveguide with flange dimensions of $50 \mathrm{~mm}$ [19]. According to the results obtained in the previous section, two fixed thicknesses of the test material were used of $1 \mathrm{~mm}$ and $5 \mathrm{~mm}$ respectively. In this analysis, these two thicknesses were assumed to be fixed for each one of the four considered cases. Also, it was assumed that each one of the two known materials has the same thickness and they vary equally from the minimal thickness of $2 \mathrm{~mm}$ to the largest possible value. Table 3 lists the details of the four considered cases of the structure in the FDTD simulations.

Table 2. The FDTD results of complex reflection coefficient compared with analytical [11] and experiment results for single-layer and two-layer methods at $f=10 \mathrm{GHz}$

\begin{tabular}{cccc}
\hline Method & Model & $|\Gamma|$ & $\Gamma_{\text {phase }}$ (in Deg.) \\
\hline \multirow{2}{*}{ Single-layer } & FDTD & 0.5058 & -134.220 \\
& Analytical [11] & 0.5111 & -133.731 \\
& Experiment & 0.5102 & -132.987 \\
Two- layer & FDTD & 0.4793 & -140.349 \\
& Analytical [11] & 0.4865 & -139.620 \\
& Experiment & 0.4811 & -138.479 \\
\hline
\end{tabular}




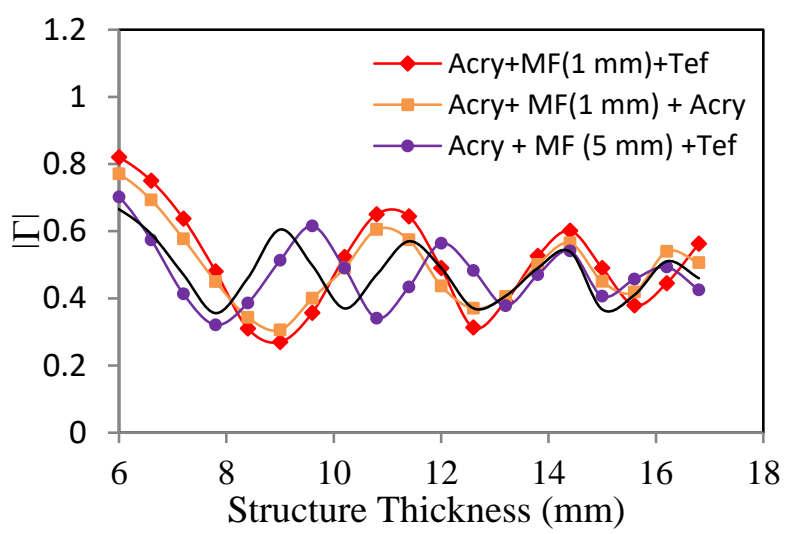

Figure 4. Variations of reflection coefficient magnitude with thickness of the three-layer structure

The results of variations of probe reflection coefficient magnitude for the considered cases obtained at $10 \mathrm{GHz}$ are shown in Fig. 4. From the figure, the following can be observed. Firstly, for the two thicknesses of the test material, the variations in the magnitude of probe reflection coefficient in case of using two identical layers of known materials (Acrylic) are relatively less than that obtained in case of using two different layers of the known materials (Acrylic and Teflon). This is due to the fact that Acrylic has a higher value of loss factor than that of Teflon. Secondly, it can be observed that using $5 \mathrm{~mm}$ thickness of each one of known materials is reasonable to obtain better approximation of reflection coefficients measurement accuracy for the specified thickness range of the test material (from $1 \mathrm{~mm}$ to $5 \mathrm{~mm}$ ). Lastly. for the four considered cases, the reflection coefficient magnitude variation has a behavior of oscillatory-damped decreasing with the thickness of the structure (for fixed thickness of test material). The obtained results of this analysis show that in order to provide two different conditions of testing, the proposed structure should be arranged such that the test material is sandwiched between two identical low-loss material (either Teflon and Teflon or Acrylic and Acrylic) to obtain the first condition of testing (first reflection coefficient). The other condition of testing can be obtained when the test material is sandwiched between two different lowloss materials to obtain the second reflection coefficient. These two arrangements of the three-layer structure are necessary so that the probe reflection coefficient can be partly changed to carry different information and they can be distinguished by the measuring instruments. The results of this analysis are useful in the measurement setup designing requirements of $\varepsilon_{\mathrm{r}}$ and $\mu_{\mathrm{r}}$ estimation accuracy.

\section{EXPERIMENTAL VERIFICATION}

\subsection{Experimental Results}

The conclusions based on FDTD analysis of the proposed technique were experimentally verified to determine $\varepsilon_{\mathrm{r}}$ and $\mu_{\mathrm{r}}$ of radar absorbing materials using the measurement setup depicted in Fig. 5. The setup of

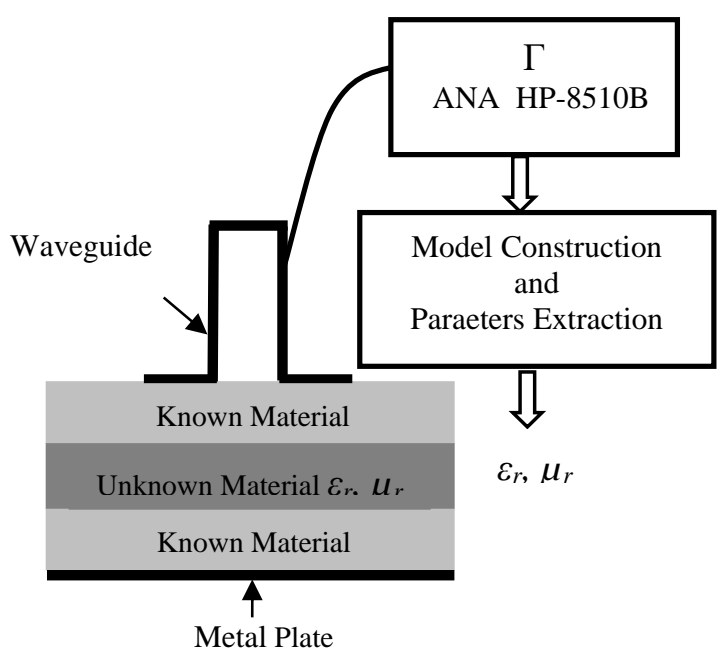

Figure 5. Measurement setup 
the measurement is divided into two parts. The first part is hardware-oriented and consisting of X-band rectangular waveguide probe placed on the top of the layered structure and then, with automatic network analyzer (ANA) used for reflection coefficient measurement. The second part is the software-oriented conversion using FDTD modeling by which the reflection coefficient is calculated. Once the FDTD modeling is performed, the main problem left is EM parameters extraction from the measured and calculated probe reflection coefficients using iterative optimization technique algorithms.

A series of experiments were carried out using rectangular waveguide probe with flange dimension of $50 \mathrm{~mm}$ to extract both complex permittivity $\varepsilon_{\mathrm{r}}(\mathrm{f})=\left(\varepsilon^{\prime}-j \varepsilon^{\prime \prime}\right)=\varepsilon^{\prime}\left(1-\mathrm{j} \tan \delta_{\varepsilon}\right)$ and complex permeability $\mu_{\mathrm{r}}(\mathrm{f})=\mu^{\prime}-$ $\left.j \mu^{\prime \prime}\right)=\mu^{\prime}\left(1-j \tan \delta_{\mu}\right)$ of several radar absorbers. The first test was performed on radar absorber with thickness of $2.4 \mathrm{~mm}$. The known backing materials used were Acrylic and Teflon with thickness of $5 \mathrm{~mm}$ respectively. The measurement was performed at a frequency of $9.3 \mathrm{GHz}$. Table 4 shows the measured $\varepsilon_{\mathrm{r}}$ and $\mu_{\mathrm{r}}$ results of the test material compared with previously published data in [11]. It is clear that the obtained results of $\varepsilon_{\mathrm{r}}$ and $\mu_{\mathrm{r}}$ and the reference data are fairly in close agreement. The small discrepancy in the obtained results may be due to the way by which the problem geometry in [11] is modeled to calculate the probe reflection coefficient. It is to be noted that the reference data are obtained using reflection-transmission method. Another set of measurements was conducted to evaluate the proposed technique performance to extract $\varepsilon_{\mathrm{r}}$ and $\mu_{\mathrm{r}}$ compared to the results obtained using single-layer method and two-layer method. The test material used in these tests was Eccosorb MF-116 radar absorber with $1.4 \mathrm{~mm}$ thickness. For single-layer method, FVM was used with frequency interval of $0.4 \mathrm{GHz}$ to obtain the needed two reflection coefficients. For the two-layer method Acrylic as known backing material is used with $8 \mathrm{~mm}$ thickness. Table 5 lists the results of $\varepsilon_{\mathrm{r}}$ and $\mu_{\mathrm{r}}$ measurement of the three methods obtained at a frequency of $10 \mathrm{GHz}$ compared to the reference data [11]. It is obvious from the table that, in comparison with the results of the single-layer and two-layer methods, the measured values of $\varepsilon_{\mathrm{r}}$ and $\mu_{\mathrm{r}}$ using the proposed technique agreed well with the published data.

Table 3. The details of the four considered cases in the FDTD simulations of the layered structure

\begin{tabular}{ccccc}
\hline \multirow{2}{*}{ Case } & Test Material & \multicolumn{3}{c}{ Three-layer Structure } \\
\cline { 3 - 5 } & Thickness $(\mathrm{mm})$ & Layer 1 & Layer 2 & Layer 3 \\
\hline$\# 1$ & 1.00 & Acrylic & MF-116 & Teflon \\
$\# 2$ & 1.00 & Acrylic & MF-116 & Acrylic \\
$\# 2$ & 5.00 & Acrylic & MF-116 & Teflon \\
$\# 3$ & 5.00 & Acrylic & MF-116 & Acrylic \\
\hline
\end{tabular}

Table 4. Comparison of the measured results of $\varepsilon_{\mathrm{r}}$ and $\mu_{\mathrm{r}}$ of radar absorber using the proposed technique with the published data [11] at $f=9.3 \mathrm{GHz}$

\begin{tabular}{lcccc}
\hline \multirow{2}{*}{ Test Method } & \multicolumn{4}{c}{ Parameters } \\
\cline { 2 - 5 } & $\varepsilon^{\prime}$ & $\tan \delta \varepsilon$ & $\mu^{\prime}$ & $\tan \delta \mu$ \\
\hline Proposed Technique & 11.38 & 0.016 & 1.50 & 0.58 \\
Published Data [11] & 11.5 & 0.02 & 1.48 & 0.60 \\
\hline
\end{tabular}

Table 5. Results of $\varepsilon_{\mathrm{r}}$ and $\mu_{\mathrm{r}}$ using different methods at $f=10 \mathrm{GHz}$

\begin{tabular}{ccccc}
\hline & \multicolumn{4}{c}{ Parameters } \\
\cline { 2 - 5 } Test Method & $\varepsilon^{\prime}$ & $\tan \delta \varepsilon$ & $\mu^{\prime}$ & $\tan \delta \mu$ \\
\hline Single-Layer Method & 15.63 & -0.028 & 1.57 & 0.71 \\
Two-Layer Method & 16.39 & 0.015 & 1.54 & 0.74 \\
Proposed Technique & 16.07 & 0.037 & 1.48 & 0.69 \\
Reference Data [11] & 16.0 & 0.06 & 1.50 & 0.68 \\
\hline
\end{tabular}


For all methods considered in this work, the purpose of using the metal plate for backing the test material is to increase measurement sensitivity. However, the obtained results have shown that backing of test material by the metal plate influences the measured values of $\varepsilon_{\mathrm{r}}$ and $\mu_{\mathrm{r}}$. In the case of single layer method, since the test material is directly backed by metal plate, the magnetic field in the test material expected to be strong resulting in fairly accurate measurement of $\mu_{\mathrm{r}}$. On the other hand, the results of $\varepsilon_{\mathrm{r}}$ measurement are highly influenced by the presence of the metal plate, which yields in reduction of the electric field interrogation inside the test material region [7]. This is also true for the two-layer method, but the result of $\varepsilon_{\mathrm{r}}$ measurement is less influenced since the test material is separated from the probe flange by low-loss material. For the proposed technique, in comparison with the reference data, a reasonable accurate $\varepsilon_{\mathrm{r}}$ result is obtained due to separation of the test material from the metal plate by low-loss material resulting in a reasonable interrogation of both electric field and magnetic field with the test material. Also, the first layer of low loss material, which separates the test material and probe flange, contributes to decrease the influence of both radial and surface waves. This arrangement makes both of them greatly depressed by the structure. In general, in comparison with two-layer method results, the experimental results of the proposed technique show a reasonable improvement obtained in the measured values of both $\varepsilon_{\mathrm{r}}$ and $\mu_{\mathrm{r}}$. Another comparison was made between the measured results of both $\varepsilon_{\mathrm{r}}$ and $\mu_{\mathrm{r}}$ using the proposed technique and the results obtained using two-layer method over a given frequency range. The test material used was Eccosorb MF-116 radar absorber with $2.8 \mathrm{~mm}$ thickness. Frequency-sweep measurement technique is employed [20] to perform broadband measurement process over a given frequency range by which the needed independent reflection coefficients are measured in one step. Consequently, the technique speeds up and simplifies the process of measurement and decreases repeatability and error that may occur if other methods are used. Thus, the accuracy of the measurement can be improved.

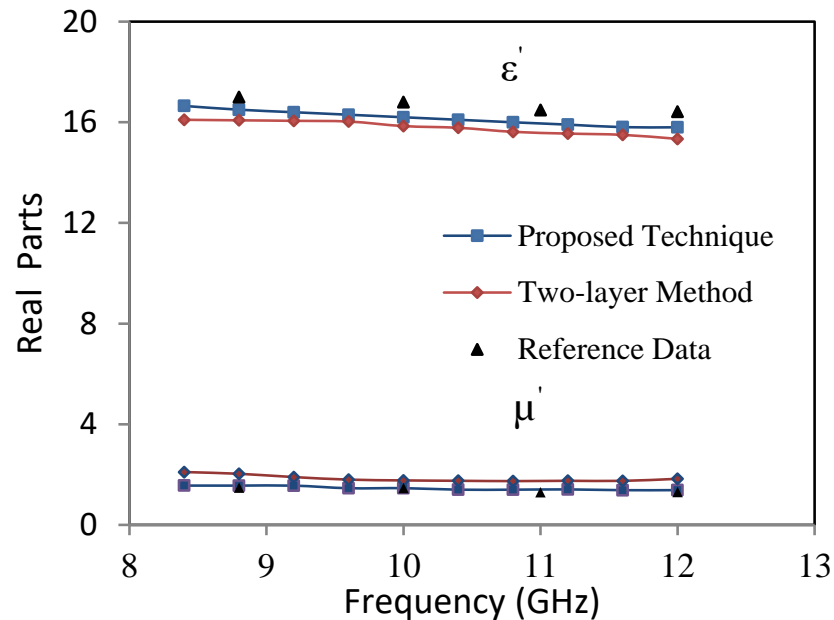

(a)

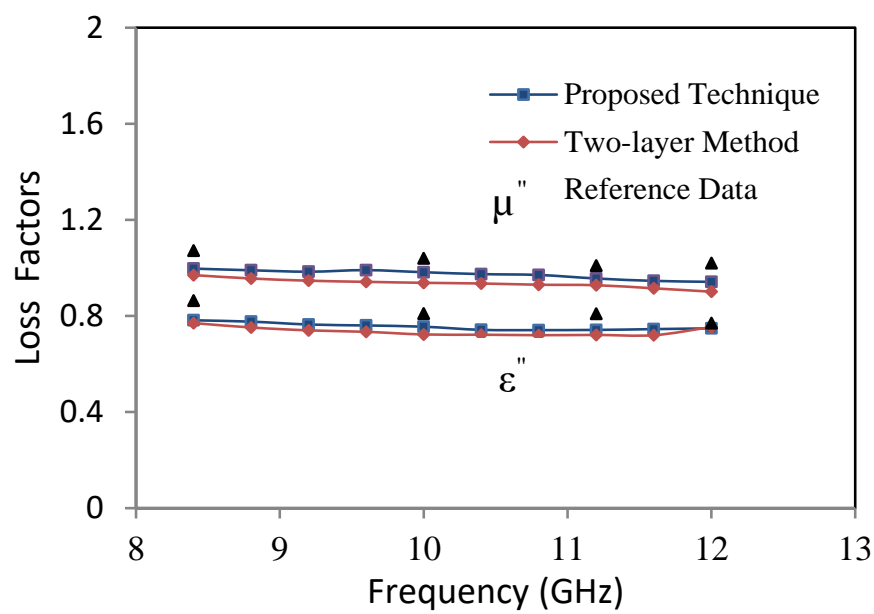

(b)

Figure 6. The results of the measured complex permittivity and complex permeability of MF 116 radar absorber using the proposed technique and two-layer method 
Ten frequency points were selected with frequency interval of $0.4 \mathrm{GHz}$ between two frequency points and the results are shown in Fig. 6. The real parts of the measured $\varepsilon_{\mathrm{r}}$ and $\mu_{\mathrm{r}}$ are shown in Fig. 6(a) and the loss factors are shown in Fig. 6(b). The inspection of the results presented in the two figures reveals that the proposed technique geometry improves the results of both $\varepsilon_{\mathrm{r}}$ and $\mu_{\mathrm{r}}$ compared to the results obtained using two-layer method geometry. It is clear that the results of $\varepsilon_{\mathrm{r}}$ and $\mu_{\mathrm{r}}$ obtained using the technique compared with reference data is much better than the results obtained using two-layer method. The obtained results imply that backing of test material directly by metal plate do not yield an accurate in the measured $\varepsilon_{\mathrm{r}}$ and $\mu_{\mathrm{r}}$ especially $\varepsilon_{\mathrm{r}}$ results of tested material. On the other side of the proposed technique, the separation of the test material from the metal plate by low-loss material provides a considerable electric field interrogation, which yield more accurate $\varepsilon_{\mathrm{r}}$ results.

\subsection{Extraction of Material Properties}

For material characterization using different methods, inverse problem is used to retrieve or estimate any one of the electrical or physical properties from the measured and theoretical reflection coefficients of the probe. This is due to the fact that, for the theoretically developed expressions of probes, a closed form does not exist to retrieve or extract these parameters in terms of reflection coefficients. For multilayer structure, the unknown parameters can be any one from the set of $\left\{\varepsilon_{r l}, \mu_{\mathrm{rl}}\right.$ and $\left.d_{l}\right\}$, where $l=1,2,3, \ldots, L$ is the structure layers number. Thus, it becomes necessary to extract them by numerical inversion from theoretical expressions developed for probe reflection coefficient [21]. Hence, to simultaneously measure complex permittivity and complex permeability, two complex reflection coefficients are needed. Basically, the numerical inversion is performed to extract them by imposing the measured reflection coefficient $\left(\Gamma_{\text {meas }}\right)$ to theoretically calculated one $\left(\left(\Gamma_{\text {thy }}\right)\right.$ using $(17)$.

$$
\Gamma_{\text {meas }}\left(f, d, \varepsilon_{r}, \mu_{r}\right)-\Gamma_{\text {thy }}\left(f, d, \varepsilon_{r}, \mu_{r}\right) \leq \delta
$$

from which the unknown parameters of the test material can be retrieved iteratively by using search algorithms, where $\delta$ is the error in the measurement. It is apparent from (17) that accurate extracted values of $\varepsilon_{\mathrm{r}}$ and $\mu_{\mathrm{r}}$ are determined in part to accurately predict the theoretical reflection coefficient. In the most of the theoretical formulations, the probe reflection coefficients are developed under assumption that probe flange is infinitely large. This assumption may produce errors in the estimated values of both $\varepsilon_{\mathrm{r}}$ and $\mu_{\mathrm{r}}$ due using probe with finite size flange.

For the proposed technique, parameters of the test material $\left(\varepsilon_{\mathrm{r}}\right.$ and $\left.\mu_{\mathrm{r}}\right)$ can be extracted from both the measured reflection coefficients and the calculated ones when the differences between them are minimized using (18).

$$
\left\{\begin{array}{l}
\Gamma_{1}^{\text {meas }}\left(f, d_{2}, \varepsilon_{r 2}, \mu_{r 2}\right)-\Gamma_{1}^{\text {calc }}\left(f, d_{1}, d_{2}, d_{3}, \varepsilon_{r 1}, \mu_{r 1}, \varepsilon_{r 2}, \mu_{r 2}, \varepsilon_{r 1}, \mu_{r 1}\right) \leq \delta \\
\Gamma_{2}^{\text {meas }}\left(f, d_{2}, \varepsilon_{r 2}, \mu_{r 2}\right)-\Gamma_{2}^{\text {calc }}\left(f, d_{1}, d_{2}, d_{3}, \varepsilon_{r 1}, \mu_{r 1}, \varepsilon_{r 2}, \mu_{r 2}, \varepsilon_{r 1}, \mu_{r 1}\right) \leq \delta
\end{array}\right.
$$

The system of equations described by (18) can be solved iteratively using the Newton-Raphson method or Levenberg-Marquardt method to within a tolerance $\delta=10^{-6}$ [22]. By guessing good initial values for both $\varepsilon_{\mathrm{r}}$ and $\mu_{\mathrm{r}}$, the two methods guarantee to be convergent in most cases. In general, it is found that the convergence of the numerical inversion is sensitive to both thickness of test material and measurement error of reflection coefficient. For a fixed test material thickness, the convergence stability may be branched off over the range of given frequency especially in multilayer structure problems. To improve the convergence of extraction process and reach the desired solution, an optimum cost function $(F)$ was employed using (19):

$$
\begin{aligned}
F\left(f_{i}, \varepsilon^{\prime}, \varepsilon^{\prime \prime}, \mu^{\prime}, \mu^{\prime \prime}, d_{2}\right) & =\left[\Gamma_{\text {meas }}\left(f_{i}, \varepsilon_{2}^{\prime}, \varepsilon_{2}^{\prime \prime}, \mu_{2}^{\prime}, \mu_{2}^{\prime \prime}, d_{2}\right)\right. \\
& \left.-\Gamma_{\text {calc }}\left(f_{i}, \varepsilon_{1}^{\prime}, \varepsilon_{1}^{\prime \prime}, \mu_{1}^{\prime}, \mu_{1}^{\prime \prime}, \varepsilon_{2}^{\prime}, \varepsilon_{2}^{\prime \prime}, \mu_{2}^{\prime}, \mu_{2}^{\prime \prime}, \varepsilon_{3}^{\prime}, \varepsilon_{3}^{\prime \prime}, \mu_{3}^{\prime}, \mu_{3}^{\prime \prime}, d_{1}, d_{2}, \mathrm{~d}_{3}\right)\right]^{2}
\end{aligned}
$$

For efficiently employing the iterative search algorithms, the given cost function in (19) required to be_minimized for the measurement diversity over a given range of frequency band using (20) [9]:

$$
\begin{aligned}
F\left(f_{i}, \varepsilon^{\prime}, \varepsilon^{\prime \prime}, \mu^{\prime}, \mu^{\prime \prime}, d_{2}\right) & =\frac{1}{N} \sum_{i=1}^{N}\left(\left[\Gamma_{\text {meas }}\left(f_{i}, \varepsilon_{2}^{\prime}, \varepsilon_{2}^{\prime \prime}, \mu_{2}^{\prime}, \mu_{2}^{\prime \prime}, d_{2}\right)\right.\right. \\
& \left.\left.-\Gamma_{\text {calc }}\left(f_{i}, \varepsilon_{1}^{\prime}, \varepsilon_{1}^{\prime \prime}, \mu_{1}^{\prime}, \mu_{1}^{\prime \prime}, \varepsilon_{2}^{\prime}, \varepsilon_{2}^{\prime \prime}, \mu_{2}^{\prime}, \mu_{2}^{\prime \prime}, \varepsilon_{3}^{\prime}, \varepsilon_{3}^{\prime \prime}, \mu_{3}^{\prime}, \mu_{3}^{\prime \prime}, d_{1}, d_{2}, \mathrm{~d}_{3}\right)\right]^{2}\right)
\end{aligned}
$$


Where $N$ is discrete frequency points. The local minima number is significantly reduced using this function.

\section{THCKNESS INFLUENCE ON EM PARAMETER MEASUREMENT}

The ability of radar absorbers for electromagnetic waves absorption is determined by the specified values of both $\varepsilon_{\mathrm{r}}$ and $\mu_{\mathrm{r}}$, operating frequency $(f)$ and material thickness $(d)$. It is clear from (1) that although the measurement frequency is the independent variable, it has been shown that, both $\varepsilon_{\mathrm{r}}$ and $\mu_{\mathrm{r}}$ for a variety of practical solid material are frequency dependent, and for the most cases they are slowly varied with frequency [23-24]. On the other hand, test material thickness is another factor influencing the accuracy of the measured values of these parameters. It becomes necessary, in this regard, to investigate the behavior of high-loss materials EM properties with frequency for a range of test material thickness using the proposed technique. A series of measurements were carried out to evaluate the influence of test material thickness on $\varepsilon_{\mathrm{r}}$ and $\mu_{\mathrm{r}}$ measurement accuracy over frequency range of $(8.2-12.4 \mathrm{GHz})$. The test material used for this purpose was a composite ferrite absorber with two thicknesses selected as $2.08 \mathrm{~mm}$ and $6.24 \mathrm{~mm}$ respectively. Frequency-sweep technique was employed using eleven frequency points selected over the given frequency range with a frequency interval of $0.4 \mathrm{GHz}$ between two frequency points.

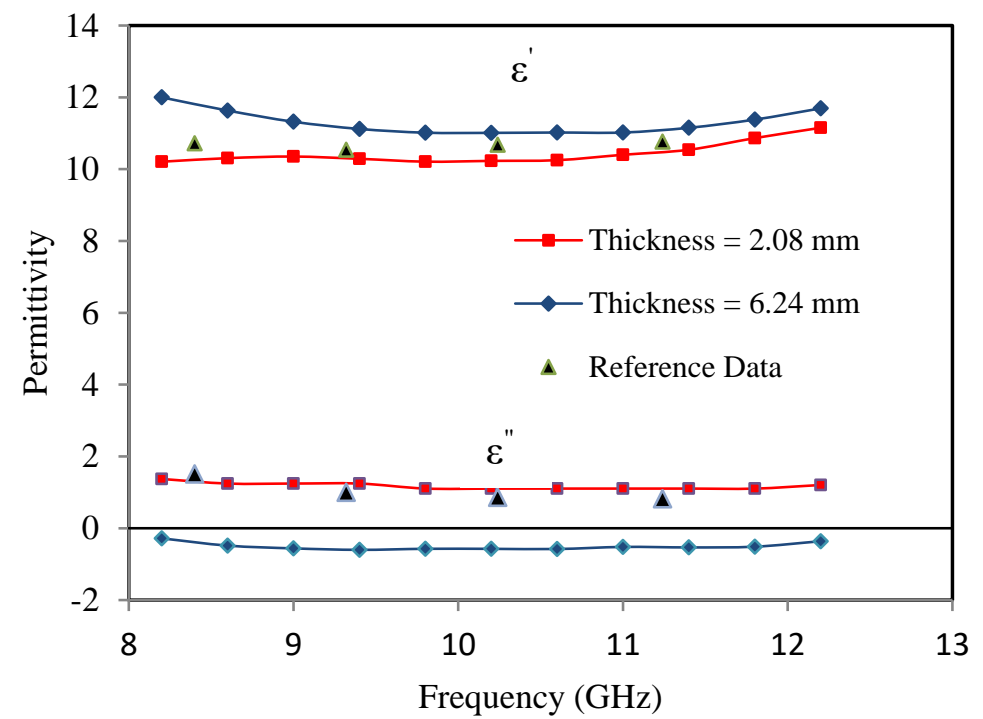

(a)

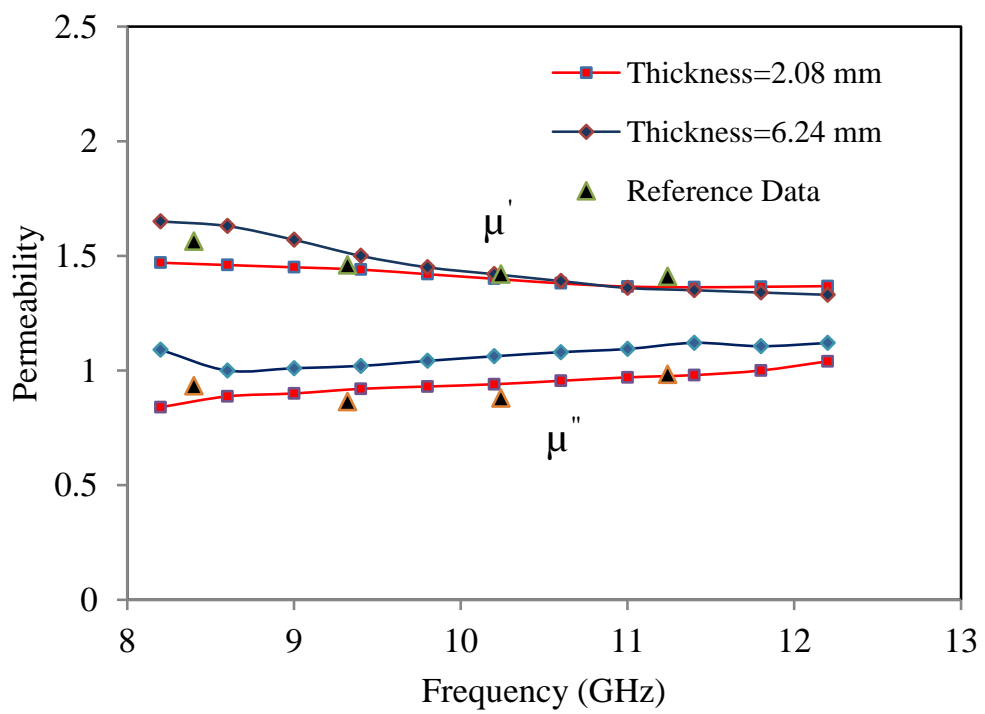

(b)

Figure 7: Variations of complex pemittivity $\left(\varepsilon_{\mathrm{r}}\right)$ and complex permeability $\left(\mu_{\mathrm{r}}\right)$ with frequency and test material thickness (a) Permittivity (b) Permeability 
The results compared with reference data are presented in Fig. 7. The variations of the real part as well as the loss factors for both permittivity and permeability are shown in Fig 7 (a) and Fig. 7 (b) respectively. Good agreement is obtained between the $\varepsilon_{\mathrm{r}}$ and $\mu_{\mathrm{r}}$ measured results and the data provided by fabricator for $2.08 \mathrm{~mm}$ thickness sample while a large discrepancy is observed between them for the sample with $6.24 \mathrm{~mm}$ thickness. Also, it can be observed that the loss factor of the permittivity becomes minus. Physically, this yield meaningful results. The results show that the sample thickness is the dominant reason such that the $6.24 \mathrm{~mm}$ test material sample thickness is too thick and such that the wave reflected from the metal plate is greatly decayed, which in turn cannot influence the input wave at the aperture as compared to $2.08 \mathrm{~mm}$ sample thickness results. Moreover, the reflection-only waveguide probe, at its best, is to be used for high-loss material testing. Hence getting a reasonable accuracy is quite difficult for testing materials with a loss factor value less than 0.1 (the loss factor value for the used sample is 0.062 ). The obtained results of $\varepsilon_{\mathrm{r}}$ and $\mu_{\mathrm{r}}$ for the two considered cases of test material thickness showed that the measurement accuracy of the thick materials with low-loss factor becomes poor. Therefore, to ensure the required measurement accuracy, the test material thickness must be chosen such that a suitable reflection exists at the probe aperture depending on the nature characteristics of radar absorber to be tested. Based on the obtained measured results using the proposed technique, it was found that the optimum range of the test material thickness lies between $1 \mathrm{~mm}$ and $5 \mathrm{~mm}$ to obtain an acceptable accuracy in the measurement of $\varepsilon_{\mathrm{r}}$ and $\mu_{\mathrm{r}}$ if the known-material layers are properly chosen (both EM-parameter and thickness). For the analysis performed using the proposed technique, three main factors of error in accuracy of measurement were considered. They are the influences of direct backing of test material by metal plate, size of probe flange and test material thickness. Research work such as error analysis due to variations of test material EM-parameter and thickness, the existence of uncertainty in the thickness of the known material and improvement of inverse problem of $\varepsilon_{\mathrm{r}}$ and $\mu_{\mathrm{r}}$ extraction need to be further investigated in the future.

\section{CONCLUSION}

A multilayer structure technique was presented to improve the measured results of $\varepsilon_{\mathrm{r}}$ and $\mu_{\mathrm{r}}$ obtained using the existing two-layer method with flanged rectangular waveguide probe. A three-layer structure of test material sandwiched between two known materials in conjunction with the FDTD method was successfully employed to model and extract $\varepsilon_{\mathrm{r}}$ and $\mu_{\mathrm{r}}$ for several samples of radar absorbers. The obtained measured results have shown that the accuracy of $\varepsilon_{\mathrm{r}}$ and $\mu_{\mathrm{r}}$ of radar absorber is reasonably improved compared to that obtained using two-layer method. The flange size is another important issue contributing to the error of measurement. It has been shown that for high loss materials, using probe with definite size of the flange, is adequate for ensuring the required accuracy in the measurement. Also, It has been demonstrated that the test material thickness of test material is the main reason standing behind accurately estimating of loss factor in particular thick samples. The obtained results suggest that for accurate measurement of $\varepsilon_{\mathrm{r}}$ and $\mu_{\mathrm{r}}$, the technique can be used for high-loss materials characterization with several millimeters thicknesses. Besides improving both $\varepsilon_{\mathrm{r}}$ and $\mu_{\mathrm{r}}$ measurement accuracy, the presented technique has some advantages over the existing two-layer method. It has extension capability to be used for multiparameter measurements (simultaneously determination of $\varepsilon_{\mathrm{r}}$ and $\mu_{\mathrm{r}}$, and thickness) and for industrial on-the worksite testing of lossy layered media, which are recently encountered in potential applications.

\section{REFERENCES}

[1] I. M. D. Rosa, F. Sarasini, M. S. Sarto, and A. Tamburrano, "EMC impact of advanced carbon fiber/carbon nanotube reinforced composites for next generation erospace applications," IEEE Trans. Electromagn. Compat.,vol. 50, No. 3, pp. 556-563, 2008.

[2] M. Koledintseva, 1. Drewniak, Y. Zhang, J. Lenn, and M. Thoms, "Engineering of ferrite-based composite materials for shielding enclosures", J. Magn. Magn. Mater. (JMMM), vol. 321, pp. 730-733, 2009.

[3] L. Kong; Li, Z.; Liu, L.; R. Huang, , M. Abshinova, Z. Yang, C. Tang, P. Tan, C. Deng, S. Matitsine," Recent progress in some composite materials and structures for specific electromagnetic applications" Int. Mater. Rev. 4, pp. 203-259, 2013.

[4] F. Wang, W. Jiang, T. Hong, H. Xue, S. Gong, and Y. Zhang, "Radar cross section reduction of wideband antenna with a novel wideband radar absorbing materials," IET Microw. Antennas Propag., vol. 8, No. 7, pp. 491497, 2014.

[5] H. Ebara, T. Inoue, O. Hashimoto" Measurement method of complex permittivity and permeability for a powdered material using a waveguide in microwave band" Science and Technology of Advanced Materials 7, pp. 77-83, 2006.

[6] U. C. Hasar, "Thickness-independent Automated Constitutive Parameters Extraction of Thin Solid and Liquid Materials from Waveguide Measurements," Progress In Electromagnetics Research, Vol. 92, pp. 17-32, 2009.

[7] G. D. Dester, "Error Analysis of A two-layer method for the electromagnetic characterization of conductor-backed absorbing material using an open-ended waveguide probe," Progress In Electromagnetics Research B, Vol. 26, 121, 2010 . 
[8] M. D. Seal, M. W. Hyde and M. J. Havrilla" Nondestructive Complex Permittivity and Permeability Extraction using a Two-Layer Dual-Waveguide Probe Measurement Geometry" Progress In Electromagnetics Research, Vol. 123, pp.123-142, 2012.

[9] M. T. Ghasr, S. Devin and R. Zoughi,"Multimodal Solution for a Waveguide Radiating Into Multilayered Structures-Dielectric Property and Thickness Evaluation,” IEEE Trans. Instrum. Meas., vol. 58, No. 5, pp. 15051513, Jan. 2009.

[10] T. Tosaka, K. Fujii, K. Fukunaga, and A. Kasamatsu, "Development of complex relative permittivity measurement system based on free-space in 220-330-GHz range," IEEE Trans. Terahertz Sci. Technnol., vol. 5, No.1, pp. 102109, Jan. 2015.

[11] Maode, N., S. Yong, Y. Jinkui, F. Chrnpung, and X. Deming,"An improved open-ended waveguide measurement technique on parameters $\varepsilon$ and $\mu$ of high-loss materials," IEEE Trans.Instrum. Meas., Vol. 47, No. 2, pp. 476-481, 1999.

[12] W. Shoujun, N. Maode, and X. Deming, " A Frequency-Varying Method for Simultaneous Measurement of Complex Permittivity and Permeability with an Open-Ended Coaxial Probe" IEEE Trans Microw. Theory Tech, Vol. 46, No. 12, 1998.

[13] G. Shaopeng , W. Yongyu and J. Daiping " A Field Performance Evaluation Scheme for Microwave-Absorbing Material Coatings" Coatings, 7, 38, pp. 1-9, 2017.

[14] K. S. Yee,'Numerical solution of initial boundary-value problems involving Maxwell's equations in isotropic media", IEEE Trans Antenna Propag. AP, Vol. No. 14, pp. 302-307, 1966.

[15] G. Mur,"Absorbing boundary condition for the finite-difference approximation of the time-domain electromagnetic field equations", IEEE Trans. Eectromag. Compat EMC, Vol. 22, pp. 377-382, 1981.

[16] K. S. Kunz and R. J. Luebbers, The Finite Difference Time Domain Method for Electromagnetics, CRC Press, New York, 1993.

[17] American Society for Testing and Materials, "ASTM D5568-1: Standard Test Method for measuring Relative Complex Permittivity and Relative Magnetic Permeability of Solid Materials at Microwave Frequencies", West Conshohoken, PA:ASTM, 2001.

[18] S. Bakhtiari; S.I. Ganchev; R. Zoughi, "Open-ended rectangular waveguide for nondestructive thickness measurement and variation detection of lossy dielectric slabs backed by a conducting plate," IEEE Trans. Instrum. Meas., vol IM-42, pp. 19-24. 1993.

[19] S. Kouji, H. Osamu, and K. Ramesh," Analysis of Error Due to Exclusion of Higher Modes on Complex Permittivity Measurement Using Waveguide with Flange" IEICE Trans. Electron., Vol. 88, No 1 pp. 139- 142, 2005.

[20] A. A. Hassan, A. Modaffer and J. Sa'adee, "Frequency-sweep Technique for Broadband Thin Sheet Absorbers Characterization using Flanged Open-ended Waveguide Probe with FDTD Modeling," International Journal of Microwave and Optical Technology, vol. 14, No. 3, pp. 175-184, 2019.

[21] W. B. Weir, "Automatic measurement of complex dielectric constant and permeability at microwave frequencies," Proc. EEE, Vol. 62, No. 1, pp. 33-36, 1974.

[22] K. Madsen,H. B. Nielsen, and O. Tingle, Methods for Non-linear Least Squares Problems, Technical University of Denmark,2004.

[23] Y. He, B. Matlin, R. E. DuBroff, , J. Drewniak, and J. Zhang, " Complex Permittivity and Permeability Measurements and Finite- Difference Time-Domain Simulation of Ferrite Materials" IEEE Trans. on Electromag. Comp., vol. 52, No. 4, pp. 878-886, 2010.

[24] R. Dosoudil, E. Ušák and V. Olah, "Automated Measurement of Complex Permeability and Permittivity at high Frequencies," Journal of Electrical Engineering, Vol. 61. No 7, pp. 111-114, 2010.

\section{BIOGRAPHY OF AUTHORS}

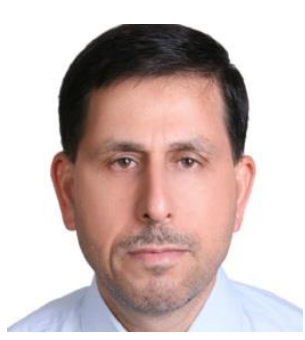

Abdulkadhim A Hassan received the B.S. Eng. degree in 1980 in Electrical Engineering (Electronics and Communications) from Mosul University, Iraq, in 1980. He then obtained his M. Eng degree (Electronics) from University of Technology Iraq, from 1981 to 1983 and Ph. D. degree (Communications and Information Engineering) from Shanghai University of Science and Technology China in 2000.

From 2000 to 2002, he was a visiting professor at United Arab Emirates University and at Ajman University of Science and Technology for the period from 2003 to 2006 . He worked as a lecturer at the Institute of Applied Technology , United Arab Emirates for the period from 2007 to 2010. From 2011 to 2013 he was a lecturer at Electronics and Communications engineering Department, College of Engineering, Kufa University, Iraq. From 2014 to 2018 he was the Head of Electrical Engineering Department, College of Engineering, Kufa University, Iraq. Since 2015, he has been an Assistant Professor in Kufa University. He is currently an assistant professor in the Department of Electrical Engineering, Faculty of Engineering, Kufa University Iraq. His research interest includes the areas of Microwave Theory and Techniques, Electromagnetic Compatibility and Microwave Measurement Techniques. He has published over 20 articles in journals and conference papers. 


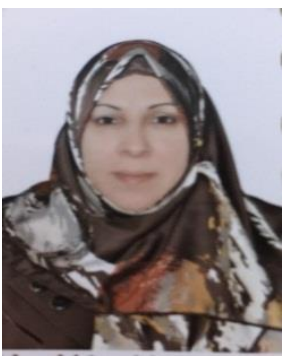

Janan H. Saadie received B. Sc. degree in 1994 from the University of Technology, Iraq, in materials science. In 2003 received the M.Sc. degree in materials science ,thin films, from the same University, Iraq. She is currently an Assistant Professor at the Department of Materials Engineering at Kufa University, Iraq. Her research interests include the areas of thin films, composite materials and nanomaterials. 